\title{
Effective Techniques to Visualize Filament-Surface Relationships
}

\author{
A. Kuß, M. Gensel, B. Meyer, V. J. Dercksen, and S. Prohaska
}

Zuse Institute Berlin (ZIB)

\begin{abstract}
Combined visualizations of filamentous structures and surrounding volumetric objects are common in biological and medical applications. Often, the structures' spatial relationships remain unclear to the viewer. In this paper, we discuss and evaluate techniques to emphasize spatial relationships. We concentrate on the visualization of transparent objects and intersecting lines. Among various techniques, participants of an exploratory user study preferred coloring of lines, marking of line-surface intersections by glyphs, and the combination of both. These techniques were additionally evaluated in a confirmatory study in which participants were asked to judge whether a filament runs through a transparent structure. We found that the evaluated techniques significantly improve the participants' performance in terms of the number of correct responses and response time. The best performance was found for the combination of line coloring and intersection glyph display.
\end{abstract}

Categories and Subject Descriptors (according to ACM CCS): Computer Graphics [I.3.6]: Methodology and Techniques - Interaction Techniques_-; Life and Medical Sciences [J.3]: Biology and Genetics-

\section{Introduction}

Visualizations of filamentous structures together with neighbouring or surrounding volumetric objects are common in biological and medical applications. For example, a neurobiologist wants to present a newly acquired insect nerve cell within a 3D brain model, or a radiologist wants to present brain nerve fibers to a patient. Questions of interest in such cases are: What trajectory does a neuronal fiber take? and Which brain regions does it connect? Does it overlap with other neurons, indicating potential connections?

Such questions could be answered by effective visualization techniques that allow a user to determine which part of a filament is contained within a particular volumetric object, where a filament enters or leaves a volumetric object, and the relative depth of filaments with respect to each other.

Illuminated tubes and transparent surface rendering are established methods, but their effectiveness for answering questions like the above is limited. Compared to simple polyline visualization, illuminated tubes provide better depth perception and differentiation. Nevertheless, understanding how the tubes are exactly embedded in space can be difficult.
The presentation of surrounding context structures can help to reduce this problem. While opaque surfaces of surrounding or neighbouring structures may occlude a large fraction of those tubes, transparent surface rendering can reduce this problem. Unlike other occlusion reduction techniques, such as cut-away views or exploded views, transparency does not hide or spatially transform any structures [Int96]. Transparency, however, has specific deficiencies. First, intersection points of tubes and transparent structures are hard to see. Second, whether a tube passes through or by a volumetric structure is often unclear.

Techniques that accentuate trajectories of lines within transparent objects could alleviate these limitations. This raises the question of which technique is most effective in this situation to support the understanding of spatial relationships. So far, user studies on line visualization methods concentrate on depth and shape perception of multiple tubes [MMYK06, WB08]. These studies demonstrate that lighting effects enhance perception. To our knowledge, the perception of filament trajectories through surrounding objects, however, has not been analyzed in a study. 


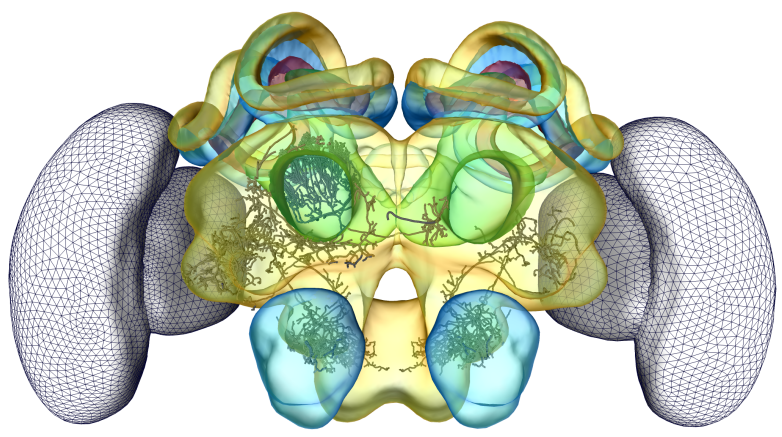

(a)

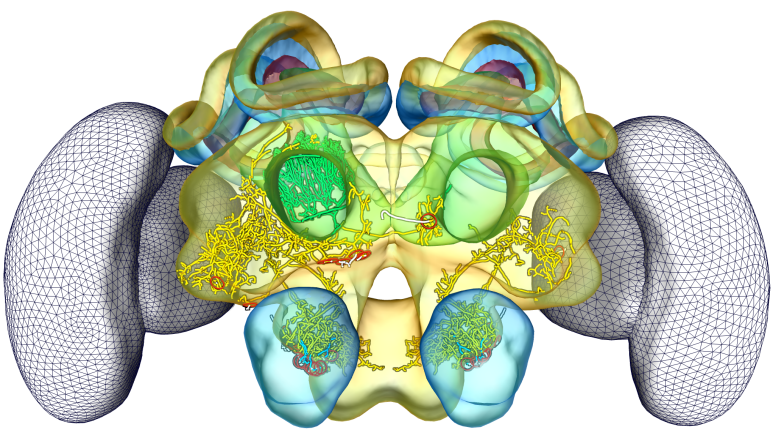

(b)

Figure 1: (a) Standard filament and surface visualization of three nerves in a bee brain. (b) The filaments are colored by their surrounding surfaces. Additionally, ring-shaped glyphs are placed at filament-surface intersections indicating, for example, registration errors. To improve the perception of which line lies in front of another, a halo effect is used.

We address this question in two steps. First, we present the results of an exploratory user study, focusing on neurobiological data (see Related Work and Section 3). The preferred techniques are the coloring of lines and the marking of line-surface intersections by glyphs. Figure 1 illustrates how these two techniques enhance a visualization. We then address the question of how effective the two preferred techniques, coloring and glyphs, are to determine whether a filament runs through an object. Section 5 presents the results of a second, confirmatory user study, in which we evaluated how well line coloring and glyphs at line-surface intersections support users to relate line segments to transparently rendered, surrounding objects.

\section{Related Work}

Filamentous structure visualization is used in several research fields, such as neuroscience [dHCMP08], flow visualization [ZSH96], vessel visualization [OP05], and DTI [MSE* 06]. In general, the developed methods try to find a compromise between speed and quality [MPSS05]. Recently, knowledge from perception theory is increasingly exploited to improve the visualizations.

The most obvious and also the fastest way to visualize filamentous structures is the rendering of simple polylines. In the fast approach of [ZSH96], polylines are illuminated to improve the perception of depth and spatial relations. Simple polylines, however, ignore possible thickness differences of the filamentous structures. Better suited for a realistic visualization is the rendering of lines using tubes or convolution surfaces [OP05]. Unfortunately, in practice these approach are often too slow. Other approaches approximate tube rendering using textured triangle strips or rectangular primitives [MSE* 06, SGS05]. They provide high visual quality and rendering speed, but artifacts can occur for lines with high curvature or at branching points. [IG97] assign different hues to line elements for a better distinction of adjacent and overlapping lines. They further use halos, which visually enhance depth discontinuities, thereby clarifying the 3D spatial organization of line elements. [BPKW03] enhance perception of spatial relations between fibers, other than relative depth, based on geometric similarity measures like end point proximity. In [WB08], it was shown that shadows improve scene layout perception as well as large-scale feature perception for scenes made up of streamtubes. [RHD*06] evaluated hatching on surfaces and hatched shadows. They conclude that both techniques improve the perception of depth and shape and the comparison of depth distances for complex tubular structures.

Presenting filament visualizations together with their surrounding or neighbouring context structures depends to a large extent on solving the occlusion problem. Techniques for occlusion reduction are, for example, cut-away views, ghosted views, and exploded views [VG05]. Unfortunately, these techniques tend to remove parts of the data, resulting in visualizations that increase the viewer's mental load. Another way to address the occlusion problem is transparency, which has the advantage of displaying all relevant structures together with unmodified shape. The primary disadvantage is that understanding the shape and location of a transparent surface is difficult, because ordinary shape and depth cues of shading and occlusion are less pronounced [IFP95]. To overcome these limitations, [IFP95] display ridge and valley lines on transparent surfaces. [DWE02] propose a view-dependent transparency rendering method based on rules defined in books for technical or scientific illustrations. In BrainGazer [BoG*09], the occlusion of surfacebased anatomical structures (including nerves) by surrounding volume-rendered image data is reduced by increasing the image transparency at spatially important surface edges.

User studies that evaluate the perceptual quality of visualization methods are becoming an important part of the visualization method development process. Studies exploring subjective parameters, such as personal preferences or taste, often use questionnaires or interviews (see [TIP05] for 
a good example that evaluates hybrid visualization methods in medicine). In other studies, participants have to perform specific tasks under varying conditions to show a method's advantage [BCFW08]. An example for a visual search task experiment is presented in [BALP09], who evaluated four emphasis techniques for structures in medical visualizations. More related to our work are the studies of [RHD*06] and [WB08]. The former showed that distance-encoded shadows and surface visualizations significantly influence the perception of spatial depth. Here, participants were asked to specify the correct order of marks on vascular structures, or to determine the depth distance order between vascular segments. [WB08] found out that global shadowing influences the spatial perception of multiple lines. They asked participants to identify whether a certain filament lies in front of another one using varying shadowing methods.

\section{Methods for Visualizing Spatial Relationships}

In this section, we propose techniques to visualize the spatial relationships between filamentous structures with potentially complex branching patterns and surrounding volumetric structures. These methods emphasize, in particular, the containment of a filament in a volumetric structure, entry and exit points and the relative depth of different filaments.

The volumetric structures are given as triangulated surfaces. A surface is closed, but not necessarily manifold. It may consist of multiple sub-surfaces called materials, each having a distinct color. The filamentous structures are given as a set of $3 \mathrm{D}$ vertices connected by piece-wise linear segments. These polylines can be connected at the branching points, thus forming a graph structure.

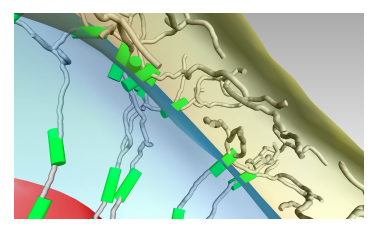

(a)

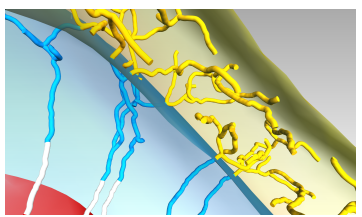

(c)

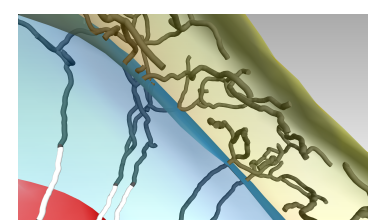

(b)

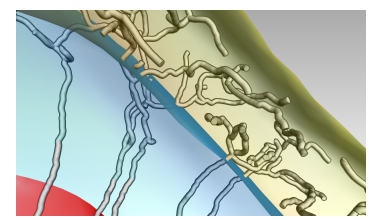

(d)
Figure 2: Examples for visualization methods. (a) Filamentsurface intersections are emphasized using green cylinders. (b) Filaments outside the surface are colored white; filaments inside are colored dark grey. (c) The filament segments are colored according to their surrounding material. (d) Depth-dependent contrast enhancement (halo).

\subsection{Standard visualization of surfaces and filaments}

We first establish a standard rendering method to which we will compare the enhanced visualization methods.

In our standard visualization, the volumetric objects are rendered as transparent surfaces. Transparency is a straightforward way to deal with occlusion in multi-object scenes, without the need to transform or discard data as with cutaway or exploded views. Additional feature lines [IFP95] are not used, because we assume that line structures on the surface as well as inside the surface are too distractive. The same applies to shadows cast by the filaments, which are not used either. Filamentous structures are displayed by illuminated tubes, which support the perception of shape and depth [MSE* 06]. Because the surfaces of our illuminated filaments are not sufficiently large, we do not place textures on them, as described in [RHD*06].

To improve the standard visualization, we evaluate the following visualization techniques:

1. Glyph-based, explicit visualization of intersection points.

2. Filament coloring according to its surrounding materials.

3. Filament color modulation according to depth.

4. Depth-based contrast enhancement.

\subsection{Glyph-based visualization of intersection points}

The explicit representation of the entry and exit points is a straightforward way to visualize whether a filament passes through a volume. To efficiently compute these intersection points, all segments and all surface triangles are inserted into an octree. For all octree leaves containing at least both a triangle and a segment, the intersection point is computed using the method described in [Eri05].

The intersection points are visualized using different types of glyphs. We propose discs and cylinders oriented along the intersecting segment (see Figure 2(a)). Alternatively, rings can be drawn around the intersection points and projected onto the intersected surface to accentuate its curvature $6(\mathrm{~b})$. This is achieved by sampling a number of points on a circle on the plane defined by the intersected triangle, and shifting each point onto the closest point on the surface. The points are then connected by computing a locally shortest path on the mesh as described in [PPP06] and [Lam08].

\subsection{Filament coloring according to surrounding material}

Color is a powerful visual feature that can be used to show differences between objects, but also to group them visually. We propose to color each filament segment according to the color of its surrounding surface. This approach visually groups filamentous and volumetric structures. We will refer to this technique as material coloring. The color of each segment in a graph of connected polylines is computed as fol- 
lows. First, the intersection points with the surfaces are computed and inserted into the polylines. Second, the color of an arbitrary end point is determined by performing a point containment query [Eri05] to find the surrounding surface and its color. Starting from the end point, the graph is traversed, switching colors at each intersection point, using the material information of the intersected triangles (see Figure 2(c)). As an alternative to material coloring, each segment can be assigned one of two colors depending on whether a segment is contained in any of the surfaces. This method, computed similarly, is called binary coloring (see Figure 2(b)).

\subsection{Filament color modulation according to depth}

In [TMC*91], depth perception is shown to be enhanced by certain color gradients. This is particularly true for saturation gradients, but also for intensity gradients. The reason is that these effects occur in natural scenes [War08]. Atmospheric scattering renders distant colors less saturated than closely viewed ones. Gradients in hue may or may not affect depth-perception. For example, a red-green hue gradient, which does not occur in natural scenes, does not affect depth perception [TMC ${ }^{*}$ 91]. On the other hand, a technique commonly used in painting is to modulate the colors of remote objects towards the background, which usually is blue [ER01]. We mimic this effect by modulating the color of all opaque objects in the scene with a depth-dependent color map. The color of transparent objects is, however, not changed. In Figure 3(c) an example is presented.

\subsection{Depth-based contrast enhancement}

To enhance the contrast for better perception of, in particular, thin filamentous structures and their relative depths, we use a halo method based on the work by [LCD06] (see Figure 2(d)). In the original method, a spatial importance function $(S I F)$ is defined as the difference between the depth buffer values and a low-pass filtered copy of the depth buffer. The SIF has large absolute values along edges where a depth difference occurs. By modulating the image color using the $S I F$, a halo-like appearance is created. A disadvantage of the original method is, however, that the filter response is skewed by background depth buffer fragments having the maximum $z$-value (1.0), which happens frequently for thin structures. In this case, the depth difference for a pair of crossing lines needs to be relatively large for a halo to appear. We, therefore, chose to leave out any background fragments when computing the filter response. Another undesired aspect of the original method is that the computed SIF-values are much smaller for far away objects than for close objects, due to the non-linear $z$-values in the depth buffer after perspective division. To increase the contrast for distant objects, the $z$-values used for computing the SIF are relinearized by inverting the perspective division.

\section{Exploratory User Study}

To obtain an indication which of the techniques presented in Section 3 support the understanding of spatial relationships, we conducted an exploratory unsupervised user study. The participants were asked to rate the effectiveness of visualizations in a printed questionnaire [Gen09].

To reduce the volume of the questionnaire, it was divided into two main parts. The first part concentrated on material coloring, binary coloring, and the additional display of intersection glyphs. For the coloring techniques, we were interested in ratings. To evaluate the potential of additional glyph display, we wanted the participants to compare the coloring techniques to the glyph display and to the combination of coloring and glyphs. In the second part, we concentrated on ratings for red-to-blue and yellow-to-red modulation according to depth and on ratings for halos.

We hypothesized:

- Material coloring is best to emphasize through which structures a filament passes, because of visual grouping of line and surface colors.

- Glyphs perform best for intersection detection since their distinctiveness draws attention exactly to these points.

- The combination of material coloring and glyphs does not perform better than their single use for trajectory and intersection detection respectively.

- Halos improve the understanding of relative filament location, since they clarify depth order at line-line crossings.

- Red-blue depth coloring improves depth perception since it adds an additional depth cue occurring in natural scenes.

\subsection{Participants}

We asked 29 participants, of whom 13 were visualizationand graphics-educated, 8 were potential users (neurobiologists), and 8 were non-experts, to fill out the questionnaire.

\subsection{Stimuli}

The stimuli were static visualizations of a bee brain model's substructures. The geometry featured several overlapping surfaces, and ramified line structures (nerve fibers). It was rendered in two ways, as overview and as closeup. Closeups were only generated for the questionnaire's first part. Apart from a standard visualization of the scene (see Section 3.1), the proposed modifications (3.2-3.5) were applied alone and in combination to create the different stimuli. Examples for different stimuli are shown in Figure 3.

\subsection{Procedure}

The questionnaire consisted of 13 tasks. Each task was composed of several questions regarding 1-4 associated stimuli. The participants were asked to rate or to compare the stimuli according to the questions.

(C) 2010 The Author(s) 


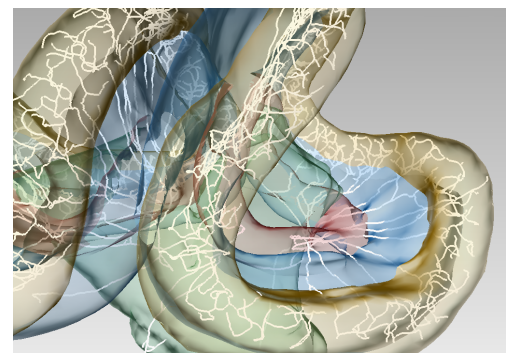

(a) Standard visualization.

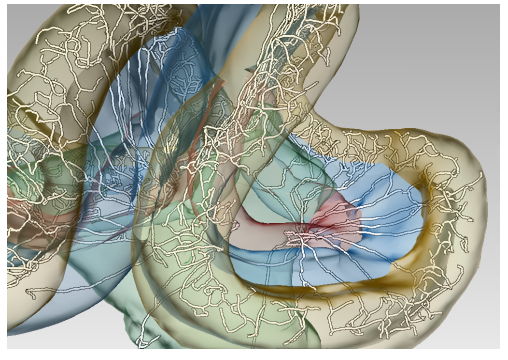

(b) Application of halo effect.

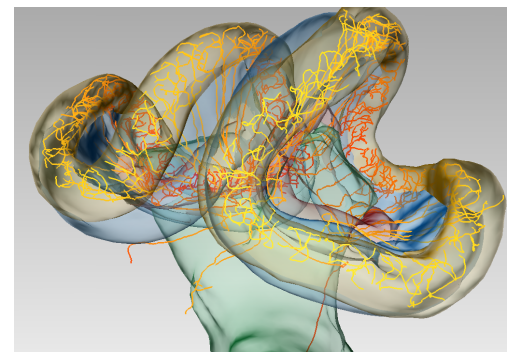

(c) Depth-coding yellow to red color gradient.

Figure 3: Example images from the first user study. Participants were asked to rate if (b), compared to (a), improves the recognition whether filaments lie behind or in front of each other. In $(c)$ they were asked to rate to the quality of depth perception.

The questions underlying the ratings or comparisons were: (1) Is the relative location of lines clear, in other words is it clear which line lies behind or in front of another line? (2) How well can you identify if lines lie inside, outside, or behind an object? (3) Is it clear where a line starts and ends and which objects it traverses? (4) How well do you recognize the intersections of lines and surfaces? (5) How well is the perception of depth? (6) How well can you estimate distances between lines? A selection of these questions was posed at each task. The questionnaire's first part concentrated on questions (1) to (4). The second part concentrated on question types (1), (2), (5), and (6).

For the rating tasks, we used overview visualizations as stimuli and presented a 6-point Likert scale (very bad to very good) for rating. For the comparison tasks a 6-point Likert scale ranging from not at all better to much better was used.

\subsection{Results}

We analyzed the rating data by testing for significant differences among the visualization techniques including the standard stimulus. Figure 5 shows the ratings for the standard visualization and the combination of material coloring and halos. The data resulting from the image comparison tasks is presented as descriptive statistical analysis. For an overview of the results see Figure 4.

\section{Rating data.}

Kolmogorov-Smirnov tests showed that not all results were normally distributed. We used the Wilcoxon signed-rank test for further analysis. We present the values $z_{(i)}$ of the $Z$ statistic below a predefined significance level $\alpha$. $(i)$ indicates the related question type (see Section 4.3).

We pairwise compared material coloring, binary coloring, and standard visualization using a Bonferroni-corrected $\alpha=0.017$. Material coloring versus standard: Material coloring of filaments received statistically significantly better ratings for all selected questions $\left(z_{(1)}=2.77, z_{(2)}=2.69\right.$, $\left.z_{(3)}=2.67, z_{(4)}=4.00\right)$. Binary coloring versus standard: Binary coloring received significantly better ratings $\left.z_{(2)}=2.36, z_{(3)}=2.39, z_{(4)}=3.71\right)$, except for understanding relative line location. Material coloring versus binary coloring: Material coloring was significantly better for relative line location and trajectory detection $\left(z_{(1)}=2.28\right.$, $\left.z_{(3)}=2.98\right)$. For the detection of intersection points and inside/outside location, the same effect could be observed, but was not significant.

Depth coloring was pairwise compared with $\alpha=0.017$. Yellow-red coloring versus standard: Yellow-red coloring significantly improved the perception of relative line location $\left(z_{(1)}=4.22\right)$ and line distance estimation $\left(z_{(6)}=2.35\right)$. Red-blue coloring versus standard: Red-blue coloring performed significantly better for relative line location $\left(z_{(1)}=3.62\right)$. Yellow-red coloring versus red-blue coloring: No colormap was significantly better than the other.

Halos versus standard: With $\alpha$ set to 0.05 , halos achieved a significantly better performance for relative line location, inside/outside line location and overall depth perception $\left.z_{(1)}=4.39, z_{(2)}=2.38, z_{(5)}=4.69\right)$.

\section{Comparison data.}

The following description lists the results of the comparison between material coloring and intersection glyphs, as well as additional benefit gained by their combined application. The comparisons were performed using both overview and closeup visualizations.

For inside/outside line location detection, $69 \%$ preferred material coloring over glyphs (17\%). This preference raised to $79 \%$ over $17 \%$ in closeups. $71 \%$ (86\% in closeups) rated the combination of both techniques to be more efficient than their single usage.

Material coloring was also preferred over glyphs for line trajectory detection $(86 \%$ versus $11 \%)$. In closeups, the ratio was $69 \%$ for material coloring versus $10 \%$ for glyphs. An additional improvement for coloring combined with glyphs has been stated by $81 \%$ and $89 \%$, respectively.

The use of glyphs was favored for the detection of intersection points by $59 \%$ of the participants whereas only $31 \%$ favored material coloring for this task. In closeups, 


\begin{tabular}{|c|c|c|c|c|c|c|}
\hline \multirow{3}{*}{$\begin{array}{r}\text { standard } \\
\text { glyphs }\end{array}$} & depth perception & in/out location & object traversal & surface intersections & relative location & line-line distance \\
\hline & 0 & 0 & 0 & 0 & 0 & 0 \\
\hline & 0 & + & + & ++ & & \\
\hline \multirow{2}{*}{$\begin{array}{l}\text { binary coloring } \\
\text { material coloring }\end{array}$} & & + & + & + & 0 & \\
\hline & & ++ & ++ & + & + & \\
\hline \multirow{4}{*}{$\begin{array}{l}\text { halos } \\
\text { yellow-red d.c. } \\
\text { red-blue d.c. } \\
\text { glyph+color }\end{array}$} & + & + & & & + & \\
\hline & 0 & 0 & & & + & + \\
\hline & 0 & 0 & & & + & 0 \\
\hline & & +++ & +++ & +++ & & \\
\hline
\end{tabular}

Figure 4: Overview of the exploratory study's rating and comparison tasks with visualization techniques in rows and question types in columns. Neglected technique-question combinations are grayed out. Os represent no improvements over the standard. The + symbols are used to indicate enhancements when applying the technique. Each time a technique was preferred, the number of + is increased. For example, regarding object traversal, binary and material coloring performed better than the standard visualization (both receiving $a+$ ); in direct comparison, material was preferred over binary coloring (leading to ++ ).

glyphs were also preferred over material coloring (55\% versus $24 \%$ ). Additional benefit by using both techniques together was observed by $67 \%$ in overview visualizations and by $93 \%$ in closeups.

\subsection{Discussion}

The results met most of our expectations. In general, the applied visualization methods improved the perception of line location and depth.

The answers differed between the groups of participants. Overall, the non-expert participants' answer variance was much higher than the variance of the other groups. They also favored the methods, but, compared to the votes of neurobiologists or graphics users, they assigned lower rankings. This may be due to the lack of experience of the participants, and the complexity of scenes and tasks.

We did not expect the combination of line coloring and intersection glyphs to be favored for all performed tasks, because we thought that glyphs might sometimes hide important information or might be distracting. However, the improvement of the combined techniques in closeups might be a sign that our thought of distraction is not completely wrong. Interestingly, the combination seems to add more to the result than its single parts. The overall very good ratings for material coloring and its preference over glyphs was surprising. Halos as well as depth-dependent color modulation received good rates. The expected preference of redblue depth coloring was not substantiated by the study.

Given these findings, we further investigated the potential of material coloring and the favored ring glyphs at intersections. We were especially interested in the combined use of both techniques. We wanted to know if the combination performs better than the single techniques for the task of judging whether a filament runs through a structure.

\section{Confirmatory User-Study}

We designed a second study to recheck and strengthen our findings. The study concentrated on the techniques that had
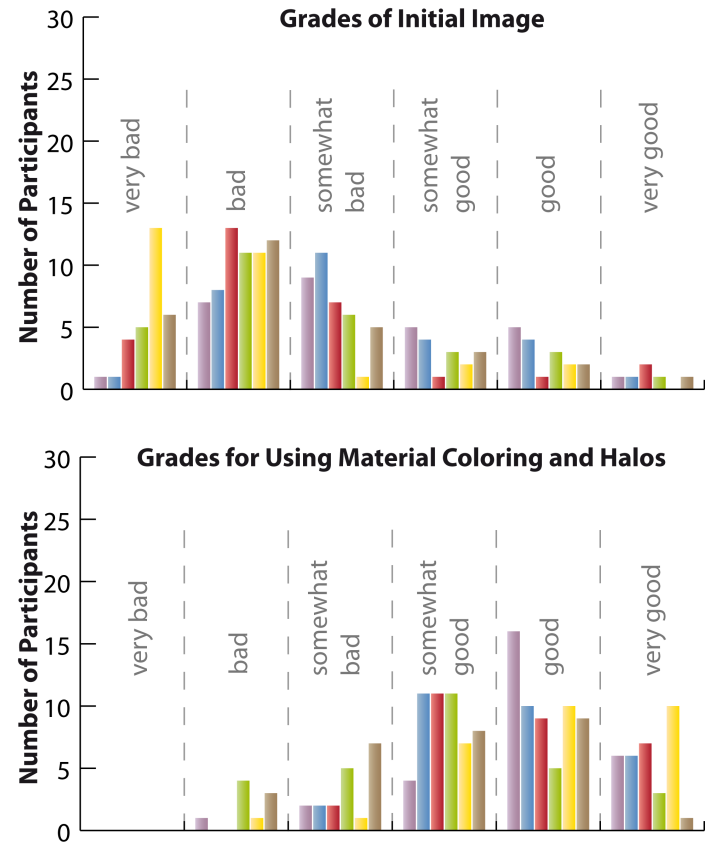

Figure 5: Visualization rating. Average rating for standard visualization (top) compared to average rating for a visualization using a combination of material coloring and halos (bottom). Bar colors represent question types from Figure 4.

been preferred in the exploratory study, namely material coloring, intersection glyphs, and the combination of both. We limited the task to identifying a line's trajectory. This reduced the complexity of the study while concentrating on a commonly performed task in neuroscience applications. We used static scenes, because we were mainly interested in the influence of the emphasizing techniques on perception. Using static scenes should also allow us to infer how suitable the techniques are for 2D media, like print or web.

Our study followed a one-factorial design in which the variations of visualization techniques were the factor's levels. They were given by a standard visualization (see Section 3.1 and Section 5.2) and visualizations that resulted 
from adding either one or both of the emphasizing techniques. Using a within-subject design, we presented different scenes of varying factor levels to our participants. In the scenes, the participants had to specify whether a filament runs through a certain structure or not. Naturally, the study's independent variables were the factor's levels. Its dependent variables were the number of correct responses and response times. Control variables were the participants' experience in visualization and computer graphics, their age, gender, and potential color vision deficiencies. Our null hypothesis $H_{0}$ stated that the techniques had no influence on the dependent variable. Our alternative hypothesis $H_{1}=\neg H_{0}$ stated that they had significant influence on the test results.

\subsection{Participants}

48 participants (17 females, 31 males) participated in our study. The participants' age ranged from 24 to 55 . Four participants had a red-green color vision deficiency. 30 percent claimed that they had much experience with visualization and computer graphics. 40 percent responded to have average experience in this field; 30 percent had little experience.

\subsection{Stimuli}

Our baseline stimuli, the first factor level, were scenes that consisted of three volumetric objects represented by surfaces and one filament, displayed in perspective projection (see Figure 6(a)). The surfaces had an organic and mostly convex shape. They had neither deep indentations nor strong concavities. We assigned different color values to the surfaces according to the color recommendations from [WGM $\left.{ }^{*} 08\right]$. To reduce the generation of false colors, we chose opposite hues from the hue wheel for the first two surfaces, specifically cyan and red. For the third surface, we chose the neutral color grey. We used transparency rendering with an opacity value of 0.3 for all surfaces. The filament was visualized as a white tube of constant diameter (approximately $\frac{1}{30}$ of the average surface object diameter). Its shape was elongated while containing several curves. The filament could intersect $0,1,2$ or all surfaces. To create visualizations for the remaining factor levels, we additionally applied intersection glyphs, material coloring, or both (Figure 6). The participants were asked: Does the filament run through the red structure?

The scenes were automatically generated. The main idea was to place the three surfaces randomly on a regular $3 \times 3 \times 3$ grid. The red target surface was always placed at the center. The first and third surface were placed in the front and back plane respectively. For each scene, three surfaces $s_{1,2,3}$ were randomly chosen from 31 different surface models. The surfaces were scaled such that the longest side of their bounding box equaled 1 . The first surface's center was translated to the origin. The two other surfaces' centers were translated in the $x y$-plane to a position randomly chosen out of the eight standard positions $\{-\delta, 0, \delta\}^{2} \backslash(0,0)$; in $z$, they were moved to $\delta$ and $-\delta$ respectively. A practical value for $\delta$ was 0.8 ; we rejected intersecting surfaces.

The filament was generated by performing a modified random walk. Starting with vertex $v_{0}$ at position $\left(3 \operatorname{sign}\left(x_{1}\right) \delta, 0,2 \delta\right)$, with $x_{1}$ being the first surface's $x$ coordinate, the filament was extended with each iteration towards the bounding box center of the next surface: $\vec{v}_{i+1}=\vec{v}_{i}+\vec{s}$. The vectorial increment $\vec{s}=\vec{d}+\vec{r}$ of each iteration was given by its directional part $\vec{d}$ and a random vector $\vec{r}$. The directional part was defined as the vector to the next bounding box center, divided by a subdivision factor that was set to 10 . The random vector was scaled to a length of $|\vec{r}|=0.7|\vec{d}|$. When the current filament vertex $\vec{v}_{i}$ was within the vicinity of the bounding box center $\vec{b},\left|\vec{b}-\vec{v}_{i}\right|<3|\vec{d}|$, the next surface's center was chosen as target. According to $v_{0}$, the filament's terminal point was placed at $\left(3 \operatorname{sign}\left(x_{3}\right) \delta, 0,-2 \delta\right)\left(x_{3}\right.$ was the third surface's $\mathrm{x}$-coordinate). Finally, we smoothed the filament.

For each factor level, we selected eight different stimuli in which the filament ran through the red structure and eight different stimuli in which it did not. This resulted in $2 \times 4 \times 8=64$ trial stimuli, each presenting a unique scene.

\subsection{Procedure}

Our testing application was a stand-alone Adobe Flashbased executable (see supplementary material for a webbased version), presenting a textual study introduction, a number of example tasks, and the actual test questions to the user. The application stored the responses and response times in a MySQL database. The testing application was presented on a 24 " LCD monitor at $1600 \times 1200$ resolution, adjusted to an aspect ratio of 4:3. The stimuli's resolution was $1136 \times 816$ pixels. Participants were seated an average of 60 $\mathrm{cm}$ away from the monitor. The testing room's light conditions were controlled by blinds.

The test started with questions about the participant's age, color blindness and visualization/computer graphics experience. After an explanation of the visualization techniques and the task, seven training runs were performed. Each task started with the presentation of a stimulus for exactly one second, followed by a screen showing the question, yes and no radio buttons, and a Continue button. The user had to select an answer with the mouse and click Continue to advance to the next question. The answering time was not limited, but we asked the participants to respond as quickly as possible. The recorded response time was the time the participant needed to select an answer and click the Continue button, starting from the moment the stimulus disappeared. During the introduction and the training session, participants were allowed to ask questions. After the training, the real experiment, consisting of 64 stimuli, started. The same 64 stimuli were presented in random order to each participant to avoid potential bias due to a particular fixed stimuli sequence. For 


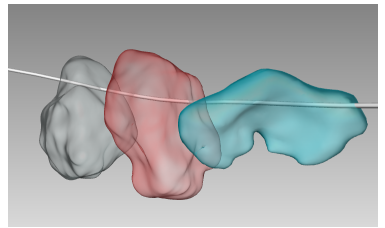

(a)

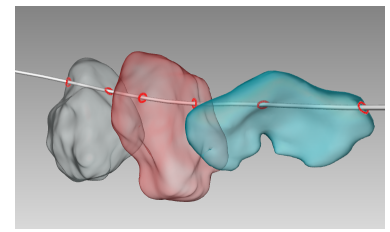

(b)

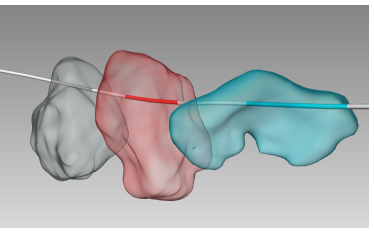

(c)

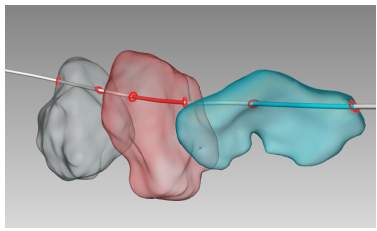

(d)

Figure 6: Example stimuli. (a) Baseline visualization. (b) Intersection points of tube and volumetric structures are displayed using rings. (c) Tube coloring by surrounding material. (d) Combined intersection display and coloring by material.

each stimulus, the participants had to decide whether the filament runs through the red structure. A complete study was performed in about 10 minutes. We tested the application with eight participants. They helped us to improve the explanation and to define the number of training runs.

\subsection{Results}

From the study, we received 3072 total trials with 768 trials for each factor level. The average number of correct responses for all trials and all participants was 52.6 of 64 ; the average response time was 2.5 seconds. We excluded the results of one participant whose number of correct responses differed more than four times the standard deviation from the mean (37 correct responses). Figure 7 shows the distribution of overall correct responses and average response times for all participants. It also presents the average number of correct responses and the average response times for each technique with their $95 \%$ confidence intervals.

Using a Kolmogorov-Smirnov test, we found that part of our data were not normally distributed. The distributions of correct responses per technique were mostly left-skewed. Cubic as well as square transformation could not normalize the data. The response times were mostly right-skewed and were, even after a logarithmic tranformation, not normally distributed. We used the non-parametric Friedman test to evaluate whether the factor visualization technique influenced the number of correct responses and the response time. To analyze each technique's influence, we performed pairwise comparisons of mean ranks according to [BLB90].

\section{Evaluation of Correct Responses}

The Friedman test showed a statistically significant influence of the visualization techniques on the number of correct responses $\left(F_{(3,138)}=111.5, p \ll 0.005\right)$. We can reject $H_{0}$ and assume $H_{1}$. Compared to the standard visualization, participants performed better when intersection glyphs, material coloring, or the combination of both (all $p \ll 0.05$ ) were used. A significant difference was also found for the combination of intersection glyphs and material coloring compared to the single use of material coloring $(p<0.05)$ and the single use of glyphs $(p \ll 0.05$ ). The effect between intersection glyph display and material coloring was not significant.

\section{Evaluation of Response Times}

The response times were significantly influenced by the different visualization techniques $\left(F_{(3,138)}=34.3, p \ll 0.005\right)$; $H_{0}$ can be rejected. The pairwise comparison showed no significant difference between the standard visualization technique and the usage of intersection glyphs. Also, the difference between material coloring and the technique combination was not significant. In contrast, we found significant differences ( $p \ll 0.05$ ) between the standard visualization and the remaining techniques (material coloring and the combination of material coloring and intersection glyphs). The difference between intersection glyphs and material coloring was also significant $(p \ll 0.05)$.

Figure 8 summarizes the results of the pairwise comparisons for correct responses and response times. Presented are the absolute differences $d$ between the rank means that resulted from the Friedman test. The critical difference $d_{\text {crit }}$ for $\alpha=0.05$ is 0.745 .

\begin{tabular}{|c|c|c|c|c|c|c|}
\hline & \multicolumn{3}{|c|}{ Response Correctness } & \multicolumn{3}{|c|}{ Response Time } \\
\hline & glyphs & color & combi & glyphs & color & combi \\
\hline \multirow{3}{*}{$\begin{array}{r}\text { standard } \\
\text { glyphs } \\
\text { color }\end{array}$} & 1.394 & 1.798 & 2.596 & 0.17 & 1.128 & 1.553 \\
\hline & & 0.404 & 1.202 & & 1.298 & 1.723 \\
\hline & & & 0.798 & & & 0.426 \\
\hline
\end{tabular}

Figure 8: Absolute mean rank differences between the stimuli variations. A value above $d_{\text {crit }}=0.745$ (highlighted in green) is statistically significant.

\section{Evaluation of Control Variables}

We also analyzed the potential influence of our control variables age, gender, color deficiency, and visualization or computer graphics experience. We found no significant influence of the control variables on the number of correct answers or the response times.

\subsection{Discussion}

Our study results show that the visualization techniques support the user in determining whether a filament runs through a transparent structure. Both accuracy and speed are improved by the techniques. The best performance is achieved for the combination of coloring and glyphs. 


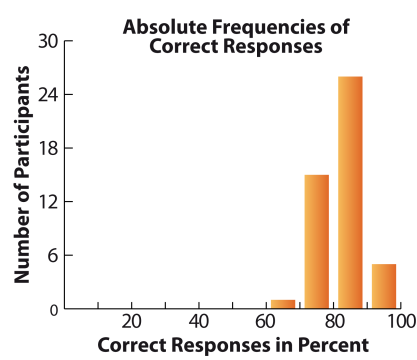

(a)

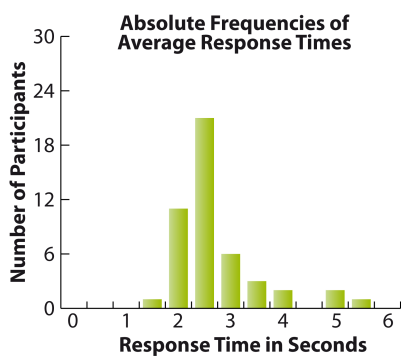

(b)

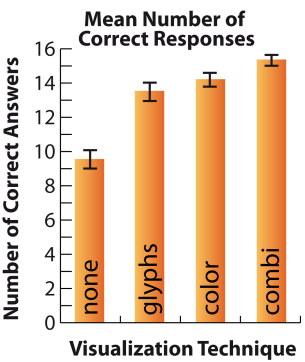

(c)

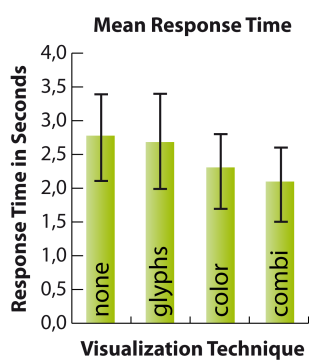

(d)

Figure 7: Number of correct responses and response times for all participants. (a) The diagram shows the absolute frequency of participants for the percentaged number of correct responses. (b) Average response times per participant ranged between 1.3 and 5.3 seconds. We show the absolute frequency of people having a certain average response time. (c) Average number of correct responses with $95 \%$ confidence intervals for each tested visualization technique. (d) Average response times together with their $95 \%$ confidence intervals for each tested visualization technique.

Some interesting observations can be made from the pairwise comparisons of the techniques. First of all, for our test stimuli, there is no significant accuracy difference between glyphs and coloring. Apparently, both visual cues allow the user to group objects and to understand spatial relationships with similar precision. The result from the exploratory study, in which the participants always preferred the coloring, could not be confirmed. However, glyphs do not help to perform the tasks faster. Compared to the standard visualization, a significant improvement of response time was only found for material coloring and the combination of techniques. This result suggests that the information conveyed by glyphs is less obvious and that it requires more time to be processed. Although there is no speedup between single coloring and the combination of techniques, the latter helps to increase the accuracy. This might be due to the increased attention that is drawn to the regions of interest by the combined techniques and the ease of grouping structures and filaments by color.

Several participants of the second study suggested to color the glyphs according to the surface they are lying on. We think that this technique variation is worth being analyzed. For our experiment, we preferred one constant eye-catching glyph color to ensure the detection of glyphs.

The material coloring technique depends on the selection of appropriate structure colors which is a difficult task. Interesting filament regions could be overlooked when the coloring is not sufficiently rich in contrast. For example, yellow on an otherwise white tube might not be detected. We chose the color values for our volumetric structures according to the color rules in [WGM $\left.{ }^{*} 08\right]$. These rules perform best for two or at most three overlapping or nested transparent structures. If more structures are used, false colors may occur.

We used highly simplified scenes for our second study. This allowed the automatic, reproducible generation of comparable images, while minimizing potential impact of scene properties that are not considered in the study design, like object geometry or number of lines, on perception. A disadvantage of this approach is that this task is simpler than real tasks in medical or biological applications. An evaluation of the techniques in more realistic scenes is desirable. Such scenes have a higher number of volumetric structures and filaments as well as branching filaments of various lengths. Questions could be how the techniques perform for different filament lengths or how the number of intersection points influences accuracy. We think that (self-)occlusion of glyphs might be a problem in scenes with a high number or densely bundled intersection points. On the other hand, we think that material coloring on its own might not be sufficiently obvious for very short traversals.

\section{Conclusion}

We evaluated several visualization techniques to improve the perception of spatial relationships between filaments and surrounding transparent structures. Two separate user studies proved some techniques to be very effective for this task. Based on the first study's results, we recommend the usage of halos and depth-based color modulation to enhance the relative depth perception of filaments. The second study showed that the correctness of recognized filament-surface traversings significantly improves when filaments are colored according to their surrounding materials and glyphs are shown at the intersection points. These techniques also accelerate the recognition time. We recommend their usage in combined visualizations of filaments and surrounding surface structures, especially in the biomedical field.

\section{Acknowledgements}

We would like to thank Edwin P. Scholte, Magic Bullet BV, Netherlands, for his technical support in creating the user study Flash application. We would also like to thank Anja Kühnlein for reviewing our statistics. Furthermore, we thank everyone who participated in the user studies. 


\section{References}

[BALP09] BAER A., AdLER F., LENZ D., PREIM B. Perception-based Evaluation of Emphasis Techniques Used in 3D Medical Visualization. In Proceedings of the Vision, Modeling, and Visualization Workshop (VMV'09) (2009).

[BCFW08] Bartz D., Cunningham D., Fischer J., WallRAVEN C.: State-of-the-Art of the Role of Perception for Computer Graphics. In Proceedings of the 29th Annual Conference Eurographics (EG 2008) (2008), pp. 65-86.

[BLB90] Bortz J., Lienert G. A., BoehnKe K.: Verteilungsfreie Methoden in der Biostatistik. Springer-Verlag Berlin Heidelberg New York, 1990.

[BoG*09] BRUCKNER S., ŁOLTÉSZOVÁ V., GRÖLlER M. E., HLAdŮVKA J., BÜHLER K., YU J., Dickson B.: BrainGazer - Visual Queries for Neurobiology Research. IEEE Transactions on Visualization and Computer Graphics 15, 6 (2009), 1497-1504.

[BPKW03] BRUN A., PARK H.-J., KnUtSSON H., Westin C.-F.: Coloring of DT-MRI Fiber Traces Using Laplacian Eigenmaps. In Proceedings of the 9th International Conference on Computer Aided Systems Theory - (EUROCAST) (2003), pp. 518-529.

[dHCMP08] De Heras Ciechomski P., Mange R., PeTERNIER A.: Two-Phased Real-Time Rendering of Large Neuron Databases. In Proceedings of the International Conference on Innovations in Information Technology (2008), pp. 712-716.

[DWE02] Diepstraten J., Weiskopf D., ERTl T.: Transparency in Interactive Technical Illustrations. Computer Graphics Forum 21, 3 (2002), 317-325.

[ER01] EBERT D., Rheingans P.: Volume Illustration: NonPhotorealistic Rendering of Volume Models. IEEE Transactions on Visualization and Computer Graphics 7, 3 (2001), 253-264.

[Eri05] ERICSON C.: Real-Time Collision Detection. Morgan Kaufmann, 2005.

[Gen09] GENSEL M.: Visualisierungsmethoden zur Verdeutlichung der räumlichen Beziehungen zwischen linien- und flächenartigen Strukturen am Beispiel neurobiologischer Daten. Diploma thesis, Freie Universität Berlin, 2009.

[IFP95] Interrante V., Fuchs H., Pizer S.: Enhancing Transparent Skin Surfaces with Ridge and Valley Lines. In Proceedings of the 6th Conference on Visualization '95 (1995), pp. 52-59.

[IG97] Interrante V., Grosch C.: Strategies for Effectively Visualizing 3D Flow with Volume LIC. In Proceedings of the 8th Conference on Visualization '97 (1997), pp. 421-424.

[Int96] INTERRANTE V.: Illustrating Transparency: Communicating the 3D Shape of Layered Transparent Surfaces via Texture. $\mathrm{PhD}$ thesis, University of North Carolina at Chapel Hill, 1996.

[Lam08] LAMECKER H.: Variational and Statistical Shape Modeling for 3D Geometry Reconstruction. $\mathrm{PhD}$ thesis, Freie Universität Berlin, Fachbereich Mathematik und Informatik, 2008.

[LCD06] LufT T., Colditz C., Deussen O.: Image Enhancement by Unsharp Masking the Depth Buffer. ACM Transactions on Graphics 25, 3 (2006), 1206-1213.

[MMYK06] MeleK Z., Mayerich D., Yuksel C., Keyser J.: Visualization of Fibrous and Thread-like Data. IEEE Transactions on Visualization and Computer Graphics 12, 5 (2006), 1165-1172.

[MPSS05] Mallo O., Peikert R., Sigg C., Sadlo F.: Illuminated Lines Revisited. In Proceedings of IEEE Visualization 2005 (2005), pp. 19-26.
[MSE*06] MERHOF D., SONNTAG M., ENDERS F, NiMSKY C., Guenther Greiner: Hybrid Visualization for White Matter Tracts using Triangle Strips and Point Sprites. IEEE Transactions on Visualization and Computer Graphics 12, 5 (2006), 1181-1188.

[OP05] Oeltze S., PREIM B.: Visualization of Vasculature with Convolution Curfaces: Method, Validation and Evaluation. IEEE Transactions on Medical Imaging 24, 4 (2005), 540-548.

[PPP06] Polthier K., Preuss E., Pinkall U.: Locally Shortest Paths on Triangle Meshes. Personal communication, 2006.

[RHD*06] RitTer F., HANSEN C., Dicken V., KonRaDVerse O., Preim B., Peitgen H.-O.: Real-Time Illustration of Vascular Structures. IEEE Transactions on Visualization and Computer Graphics 12, 5 (2006), 877-884.

[SGS05] Stoll C., Gumhold S., SeIdel H.-P.: Visualization with Stylized Line Primitives. In Proceedings of IEEE Visualization 2005 (2005), pp. 695-702.

[TIP05] Tietjen C., Isenberg T., Preim B.: Combining Silhouettes, Surface, and Volume Rendering for Surgery Education and Planning. In Proceedings of the Eurographics / IEEE VGTC Symposium on Visualization (2005), Brodlie K., Duke D. J., Joy K. I., (Eds.), Eurographics Workshop Series, pp. 303-310.

[TMC*91] Troscianko T., Montagnon R., Clerc J. L. Malbert E., Chanteau P.-L.: The Role of Colour as a Monocular Depth Cue. Vision Research 31 (1991), 1923-1390.

[VG05] Viola I., GRÖLlER M. E.: Smart Visibility in Visualization. In Proceedings of Eurographics Workshop on Computational Aesthetics in Graphics, Visualization and Imaging (2005), pp. 209-216.

[War08] WARE C.: Visual Thinking for Design. Interactive Technologies. Morgan Kaufmann, 2008

[WB08] Weigle C., Banks D. C.: A Comparison of the Perceptual Benefits of Linear Perspective and Physically-Based Illumination for Display of Dense 3D Streamtubes. IEEE Transactions on Visualization and Computer Graphics 14, 6 (2008), 1723-1730.

[WGM*08] WANG L., Giesen J., MCDONnEll K. T., ZoLLIKER P., MUELLER K.: Color Design for Illustrative Visualization. IEEE Transactions on Visualization and Computer Graphics 14, 6 (2008), 1739-1754.

[ZSH96] ZöcKler M., Stalling D., Hege H.-C.: Interactive Visualization of 3d-Vector Fields using Illuminated Stream Lines. In Proceedings of the 7th Conference on Visualization '96 (1996), pp. 107-ff. 